\title{
TUMORES DEL OVARIO
}

REVISION DE 367 CASOS ESTUDIADOS EN EL HOSPITAL SAN JUAN DE DIOS. (Cúcuta)

Por los Doctores:

Eduardo Pérez Gómez

Carlos Parada R. 粰然

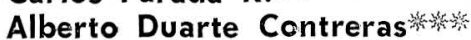
Luis Fernando Luzardo M. 深路路

\section{Introducción}

Los tumores del ovario constituyen un capítulo muy importante dentro de la patología ginecológica, y por lo tanto han sido objeto de numerosos estudios y controversias, en lo relasionado con su histogénesis, clasificación, profilaxis y tratamiento. Fueron reconocidos desde la antigüedad, pero sólamente hasta la pasada centuria se empezaron a realizar los primeros intentos quirúrgicos para su extirpación, correspondiendo al Profesor Leoncio Barreto ejecutar la primera ovariectomía exitosa en Latinoamérica el 3 de octubre de 1864 en el Hospital San Juan de Dios de Bogotá, anterior a la efectuada por Julio Clement en México en 1865 (23).

Los tumores ováricos se han encontrado en mujeres de todas las edades: la menor, un feto femenino de 30 semanas con un tumor ovárico bilateral no clasificable, y la mayor en una mujer de 92 años (12).

El cáncer ovárico es el más grave de los neoplasmas del aparato genital femenino y uno de los más serios problemas que afrontamos actualmente en la lucha anticancerosa, representando la causa principal de muerte por tumores malignos ginecológicos. Si el cáncer uterino, especialmente el de cérvix, constituye el más serio problema de salud pública en lo que respecta al sexo femenino por su elevada incidencia, el cáncer ovárico es más letal y muchísimo más difícil de diagnosticar en sus comienzos. Estadísticas recientes indican que el número de mujeres muertas de cáncer del ovario en USA se ha incrementado, habiéndose doblado en los últimos 30 años. En muchas regiones ocupa el segundo lugar entre los tumores malignos ginecológicos, superando en frecuencia al del cuerpo uterino. Representa entre el 8 y $10 \%$ de todos los cánceres genitales de la mujer, aun cuando la incidencia varía mucho por la diversidad de criterios diagnósticos, pues mientras algunos observadores clasifican como malig-

* Jefe del Departamento Gineco Obstétrico del Hospital San Juan de Dios. Cúcuta, Colombia.

** Jefe del Departamento de Patología del Hospital San Juan de Dios. Cúcuta.

*** Del Departamento Gineco Obstetrico del Hospital San Juan de Dios. Cúcuta. 
nos una gran proporción de tumores límite, otros los consideran como no malignos: igual ocurre con los tumores de células de la granulosa. En términos generales se considera que en varias regiones la incidencia de cáncer del cuerpo uterino y del ovario son similares y que alrededor del $15 \%$ de todos los tumores ováricos son malignos. El porcentaje de curación a los 5 años apenas sobrepasa el $20 \%$ a escala mundial, y se ha mantenido prácticamente estacionario en el curso de los últimos 35 años, en tanto que su mortalidad ha ido gradualmente en aumento y se ha duplicado en los últimos 25 años, no obstante los grandes adelantos en los campos de la cirugía y radioterapia, y la introducción de la quimioterapia en la terapéutica del cáncer $(8,4,12)$.

El propósito del presente estudio es hacer la revisión de los tumores del ovario en un Hospital general regional piloto, en un lapso aproximado de 13 años. No consideramos pertinente efectuar un análisis minucioso de la sintomatología, diagnóstico y tratamiento de cada grupo de neoplasias, ya que existen clásicas descripciones en los libros de texto y en múltiples trabajos.

\section{Material y Métodos}

Para el presente estudio se realizó una laboriosa revisión de los archivos del Departamento de Patología del Hospital San Juan de Dios, de Cúcuta, en un lapso de 12 años y nueve meses, comprendidos entre septiembre de 1962 y julio de 1975. Se recopilaron los protocolos de neoplasmas ováricos estudiados durante dicho período, correspondientes a pacientes hospitalarias intervenidas en el Departamento Gineco Obstétrico del Hospital, pacientes particulares de la ciudad de Cúcuta, y especímenes enviados para estudio de poblaciones circunvecinas que conforman el área de influencia del Hospital de Cúcuta: ésta abarca el Departamento Norte de Santander, la Intendencia de Arauca y población de algunas zonas limítrofes de la vecina República de Venezuela, con una extensión territorial de 44.305 kms. cuadrados y una población aproximada de 686.000 habitantes, por lo cual consideramos que los hallazgos configuran una muestra significativa en la patología tumoral ovárica de la región.

En los casos hospitalarios fue posible hacer una revisión cuidadosa de las historias clínicas correspondientes, mas no así en los particulares o remitidos para estudio, en donde los datos se entresacaron de aquellos informados por cada médico tratante en las hojas remisorias de solicitud, los cuales en muchos casos fueron exiguos e incompletos. La muestra comprendió 254 casos hospitalarios y 113 entre particulares y remitidos de otros lugares. No se consideraron los quistes no neoplásicos (foliculares, luteínicos, tecaluteínicos), variedades diversas de micropoliquistosis ovárica ni endometriosis pélvicas.

Hemos catalogado los tumores de acuerdo a la clasificación histogenética de Hertig y Gore (Cuadro № 1).

\section{CUADRO NN 1}

\section{TUMORES DEL OVARIO CLASIFICACION HISTOGENETICA (Hertig)}

\footnotetext{
1 Tumores de epitelio de revestimiento

2 Tumores de células germinales

3 Tumores de estroma gonadal específico

4 Tumores de restos embrionarios

5 Tumores del estroma inespecífico

6 Tumores metastásicos
}

El más difícil y desafiante campo de la patología ginecológica es la clasificación y entendimiento de la histogénesis de los tumores ováricos. El ovario es complejo en embriología, histología, esteroidogénesis y potencial de malignización; cada uno de 
sus tipos celulares tiene un propio potencial para formar tumor $y$, además, el ovario es sitio favorito para metástasis tumorales de muchos otros órganos. Existen grandes dificultades para establecer una clasificación que uniforme todos los criterios; aquellas basadas en la actividad hormonal del ovario afrontan serios problemas por la capacidad del órgano para sintetizar esteroides androgénicos y estrogénicos; por otra parte, la mayoría de los tumores ováricos son inertes desde el punto de vista hormonal, aunque es sugerente, también, la posibilidad de que tumores denominados inertes puedan ser endocrinológicamente activos; existen, además, lesiones funcionantes que no son verdaderos neoplasmas $(8,22)$. En los tumores ováricos, cualquier tipo celular específico no siempre produce la misma substancia hormonal, pero puede segregar varias hormonas, y también la misma hormona puede ser secretada por diferentes variedades de células.

Las clasificaciones basadas en la benignidad o malignidad de los tumores son útiles, pero no pueden ser rígidas, por las dificultades para establecer el criterio, ya que lesiones histológicamente benignas se comportan ocasionalmente como malignas en la clínica. La división entre tumores quísticos y sólidos, no es satisfactoria ya que frecuentemente se encuentran áreas quísticas y sólidas entremezcladas en un mismo tumor. El cuadro se hace más complejo por la continua aparición de un número de nombres parcial o completamente sinónimos, en la literatura. Una dificultad considerable puede encontrarse en la interpretación histológica de un tumor ovárico a causa de su gran tamaño, requiriendo un espécimen adecuado de un tedioso proceso; la interpretación de muchos tumores depende del cuidadoso estudio de secciones en múltiples sitios; en algunos casos, el mayor número de cortes hace más confuso el cuadro histológico. Con la introducción de nuevas técnicas en histoquímica, estudios enzimáticos, microscopía electrónica, citogenética, cultivo de tejidos, etc. se espera en el futuro una más profunda comprensión de la histogénesis, embriogénesis y patogénesis de los tumores ováricos (12).

\section{Resultados y Discusión \\ CUADRO № 2 \\ TUMORES DEL OVARIO HALLAZGOS EN 367 CASOS ESTUDIADOS}

\begin{tabular}{|c|c|c|}
\hline Tipo de Neoplasia & $\mathrm{N}^{0}$ Casos & $\%$ \\
\hline Cistadenoma seroso & 163 & 44.5 \\
\hline Teratoma & 126 & 34.4 \\
\hline Cistadenocarcinoma & 22 & 5.9 \\
\hline Tumor de la teca granulosa & 11 & 2.9 \\
\hline Cistadenoma seudomucinoso & 10 & 2.7 \\
\hline Carcinoma sólido & 9 & 2.5 \\
\hline Fibroma & 8 & 2.2 \\
\hline Disgerminoma & 6 & 1.7 \\
\hline $\begin{array}{l}\text { Tumores endometrioides } \\
\text { benignos }\end{array}$ & 6 & 1.7 \\
\hline Tumores de Brenner & 3 & 0.8 \\
\hline Struma ovarii & 2 & 0.5 \\
\hline Tumor de Krukenberg & 1 & 0.2 \\
\hline
\end{tabular}

Los resultados se presentan en el Cuadro № 2. De los 367 casos estudiados, 317 ( $86.9 \%$ ) correspondieron a tumores benignos, y $50(13.1 \%)$ a tumores malignos o potencialmente malignos, datos que no difieren substancialmente de los encontrados en otros estudios (12), en los cuales se dan como benignos aproximadamente el $75 \%$ de los tumores ováricos, correspondiendo la malignidad al $25 \%$ restante. No encontramos especial predilección para uno u otro ovario, aunque se dice que los disgerminomas y tumores de Brenner aparecen más comunmente en el lado derecho; $\mathrm{Fa}$ rrar y Bryant (9), no encuentran diferencia estadística significativa en 862 tumores ováricos evaluados, invo- 


\author{
G R A F I C A \\ CISTOMAS BENIGNOS
}

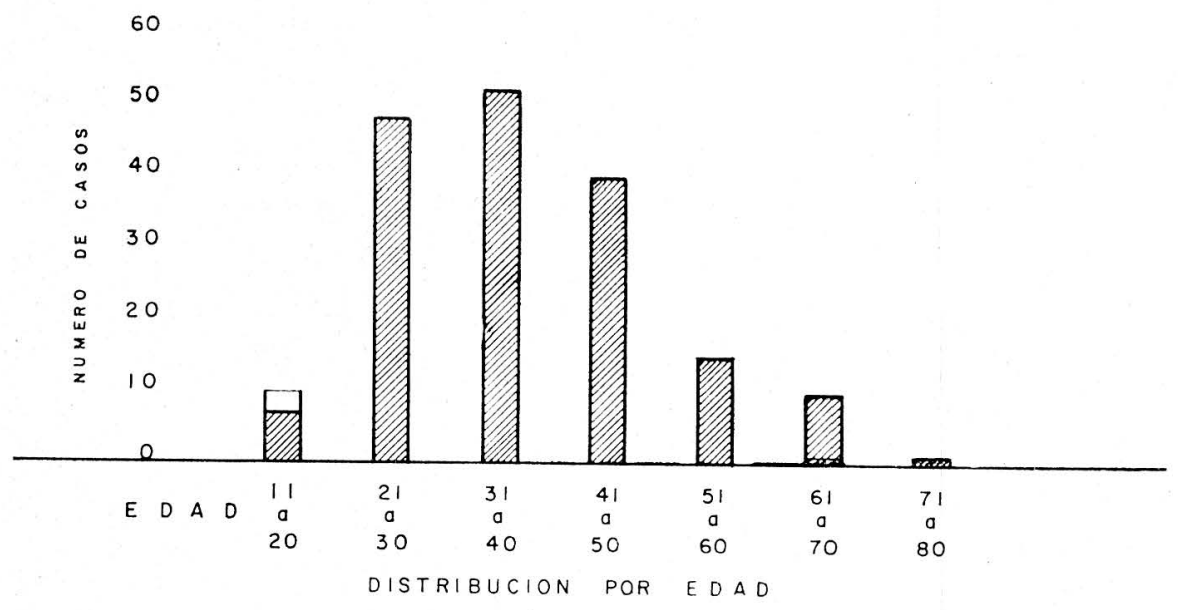

G R A F I C A I

TERATOMAS (Quiste Dermoide)

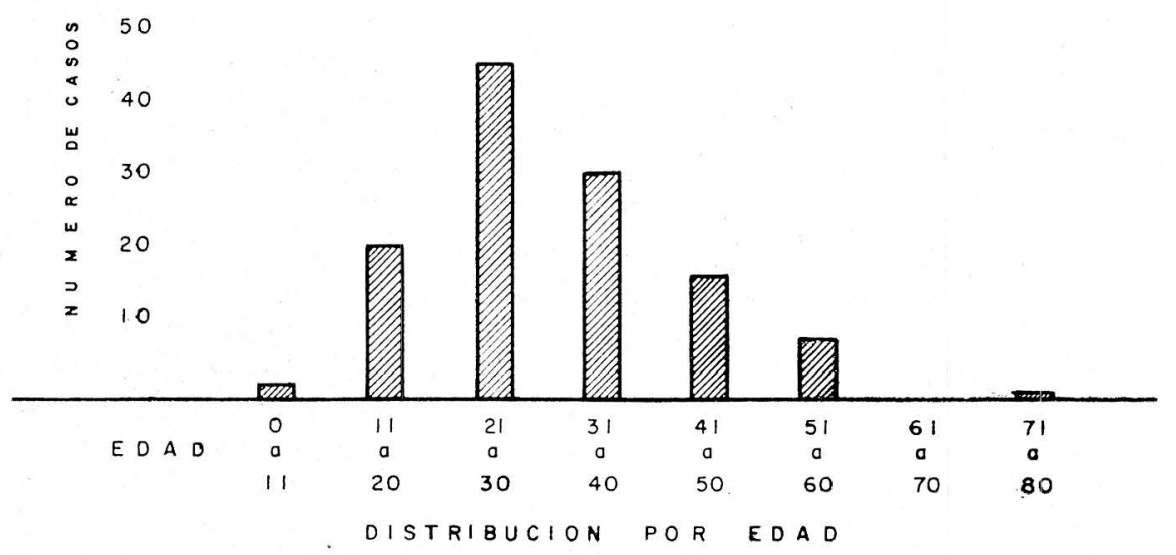


lucrando el ovario derecho o el izquierdo. La mayor incidencia de tumores benignos del ovario ocurre en el grupo de edades entre los 20 y 44 años, similar a la encontrada por nosotros y presentada en las Gráficas I y II. Quizá con aparición más temprana para los teratomas, al paso que en los tumores malignos la cúspide está entre los 45 y los 64 años, con una meclia de 53. La menor de nuestras pacientes fue una niña de 3 años con un quiste dermoide del ovario izquierdo y la mayor una anciana de 90 años con un carcinoma sólido papilar.

\section{Tumores del epitelio de revestimiento}

\section{CUADRO № 3 \\ TUMORES DEL OVARIO \\ TUMORES DEL EPITELIO DE REVESTIMIENTO}

\begin{tabular}{|c|c|c|c|c|c|c|c|c|c|}
\hline \multirow{3}{*}{$\begin{array}{l}\text { Variedad histológica } \\
\text { Cistadenoma seroso } \\
\text { Cistadenocarcinoma } \\
\text { seroso } \\
\text { Cistadenoma }\end{array}$} & \multirow{2}{*}{$\frac{N^{0} \text { Casos }}{163}$} & \multirow{2}{*}{$\frac{\text { Der. }}{78}$} & \multicolumn{2}{|c|}{ calización } & \multirow{2}{*}{$\begin{array}{c}\begin{array}{c}\text { Tamaño } \\
\text { emts. }\end{array} \\
3.26\end{array}$} & \multicolumn{2}{|c|}{$\begin{array}{c}\text { Estados } \\
\text { Embarazo }\end{array}$} & \multicolumn{2}{|c|}{$\begin{array}{l}\text { asociados } \\
\text { Torsión }\end{array}$} \\
\hline & & & 74 & 11 & & 10 & $6.1 \%$ & 22 & $13.4 \%$ \\
\hline & 20 & 6 & 6 & 8 & 8.20 & 1 & $5 \%$ & - & \\
\hline pseudomucinoso & 10 & 3 & 7 & 0 & 7.35 & - & & 3 & $30 \%$ \\
\hline $\begin{array}{l}\text { Carcinoma sólido } \\
\text { Cistadenocarcinoma }\end{array}$ & 9 & 3 & 1 & 5 & 13.25 & - & & - & \\
\hline pseudomucinoso & 2 & - & 1 & 1 & 21.32 & - & & - & \\
\hline
\end{tabular}

El mayor número fue alcanzado por los cristadenomas serosos con $163 \mathrm{ca}$ sos, en contraposición con lo descrito por varios autores que dan como más frecuente el pseudomucinoso $(4,12)$. Generalmente no alcanzan los grandes tamaños de éste porque tienen propensión a causar alteraciones secundarias de las estructuras que los rodean; cuando se descubren, por lo menos el $60 \%$ de ellos tienen más de $15 \mathrm{cmts}$. Son esferoidales, a menudo uniloculares, al superficie es lisa y brillante, aun cuando las excrecencias papilares pueden estar presentes en una alta proporción. Hay un alto porcentaje de bilateralidad que algunos estiman hasta en 35\% ( $7 \%$ de esta revisión) y su transformación maligna alcanza el $25 \%$ de los casos. Llama la atención la baja frecuencia del cistadenoma pseudomucinoso en este estudio, pero sí concuerda el dato de su gran tamaño (35 cmts. el mayor que encontramos), tamaños que alcanzan por ser generalmente asintomáticos. En Colombia fue publicado por Darnalt y colaboradores (5) un cistadenoma pseudomucinoso gigante que midió $65 \mathrm{cmts}$. y pesó 06 kgs., siendo el de mayor tamaño el publicado por Spohn (158 kg.). El promedio de bilateralidad en los casos publicados ha sido de $16 \%$, y se considera que del 5 al 10\% sufren transformación maligna (19). El epitelio mucinoso es frecuente en los teratomas ováricos habiéndose comprobado que el $5 \%$ de los quistes dermoides se asocian a cistadenomas mucinosos (24).

Dentro de los tumores malignos predominó el cistadenocarcinoma seroso (20 casos), seguido del carcinoma sólido ( 9 casos) y del pseudomucinoso ( 2 casos). Moran y Millerick (17), en una revisión de 81 casos de tumores malignos primarios del ovario, encontraron $46.9 \%$ de serosos, $13.6 \%$ de pseudomucinosos y $12.3 \%$ 
de carcinomas sólidos; su rata de supervivencia a los 5 años fue de $19.8 \%$. En la clasificación histológica de la $\operatorname{FIGO}(16,19)$ se da gran importancia como grupo a un tipo de cistadenocarcinoma ovárico primario que guarda semejanza histológica con el carcinoma endometrial; este tipo, hoy llamado "endometrioide", se consideraba antes poco frecuente; no encontramos ninguno de ellos, aunque sí un adenocarcinoma seroso asociado a un adenocarcinoma del endometrio. También uno de ellos se asoció a un embarazo, lo cual, según los reportes de la literatura, es bastante raro. Wade y colaboradores (26) en un período de 10 años encontraron un solo caso de cistadenocarcinoma pseudomucinoso asociado con embarazo, sobre un total de 40.598 gestaciones. Encontramos en un caso la asociación de un cistadenocarcinoma papilar con un tumor de células granulosas. Los carcinomas ováricos primarios bilaterales son relativamente raros. Lee (15) reporta el caso de una paciente con un carcinoma medular primario de alto grado de malignidad, localizado en el ovario izquierdo, con metástasis al derecho, y un adenocarcionma primario de bajo grado en el ova- rio derecho. El adenocarcinoma sólido del ovario puede ser una forma extrema del quiste papilar, pero más a menudo se trata de una neoplasia independiente que desde el comienzo es sólida. Rara vez alcanzan un tamaño mayor de 15 cmts.: son muy malignos, se adhieren precozmente al peritoneo pelviano y los intentos de extirpación completa son habitualmente poco exitosos.

El $65 \%$ de todos los casos de carcinoma del ovario se diagnostican por primera vez cuando el tumor es inoperable; sólamente en el $11 \%$ de los casos el tumor está localizado en un ovario y es completamente resecable cuando se descubre.

Estos datos concuerdan con nuestros hallazgos, ya que de acuerdo con la clasificación por etapas de la FIGO sólamente 5 casos ( $16 \%$ ) correspondieron al estado I, once casos al estado II (35\%), once casos al estado III $(35 \%)$ y cuatro casos al estado IV $(13 \%)$. La supervivencia del carcinoma ovárico a los 5 años es bastante baja y Maus y colaboradores reportaron en Ontario en 1968, una rata de $25 \%$ en una serie de 1.722 casos.

\section{CUADRO NN 4}

TUMORES DEL OVARIO

TUMORES DE CELULAS GERMINALES

\begin{tabular}{|c|c|c|c|c|c|c|c|c|c|}
\hline \multirow{2}{*}{$\begin{array}{l}\text { Variedad histológica } \\
\text { Teratoma }\end{array}$} & \multirow{2}{*}{$\frac{\text { No Casos }}{126}$} & \multicolumn{3}{|c|}{ Localización } & \multirow{2}{*}{$\begin{array}{c}\text { Tamaño } \\
\text { cmts. } \\
4.26\end{array}$} & \multicolumn{2}{|c|}{$\begin{array}{l}\text { Estados } \\
\text { Embarazo }\end{array}$} & \multicolumn{2}{|c|}{$\begin{array}{l}\text { asociados } \\
\text { Torsión }\end{array}$} \\
\hline & & 54 & 59 & 13 & & 12 & $9.5 \%$ & 17 & $13.5 \%$ \\
\hline Disgerminoma & 6 & 2 & 3 & 1 & 15.22 & 0 & & 0 & \\
\hline Struma ovarii & 2 & 1 & 1 & 0 & 3.7 & 0 & & 0 & \\
\hline
\end{tabular}

\section{Tumores de células germinales}

Este grupo incluye una amplia variedad, cada uno de ellos con rasgos biológicos propios, y comprenden: disgerminoma, gonadoblastoma y el grupo de los teratomas (extraembrionarios, embrionarios inmaduros y embrionarios maduros). El grupo más abundante encontrado por nosotros está constituído por el teratoma quístico maduro reconocido en la forma 
de dermoide. Además de elementos ectodérmicos, contienen formaciones plenamente evolucionadas de origen endo y mesodérmico. Hallamos 126 casos, de los cuales $10.3 \%$ fueron bilaterales' la incidencia de torsión del pedículo fue de $13.5 \%$, similar a la encontrada en los quistes serosos $(13.4 \%)$; no tuvimos ningún caso de ruptura ni de complicaciones infecciosas. En el $9.5 \%$ de los casos se asociaron a embarazo (6.1\% para los quistes serosos), aun cuando el porcentaje necesariamente se eleva si se toman solo los casos de mujeres $\epsilon$ edad reproductora: generalmente el tumor fue descubierto en el transcurso de un embarazo, con ocasión de una cesárea determinada o nó por su presencia, $O$ en el puerperio. La más joven de nuestras pacientes fue una niña de 3 años, y 9 casos ocurrieron en la postmenopausia. A los tumores ováricos corresponden aproximadamente $1 \%$ de todas las neoplasias en ginecología pediátrica; por fortuna más del $33 \%$ de tumores ováricos en niñas son teratomas quísticos benignos. Noguchi y Lonser (18) reportan un teratoma quístico benigno en una niña de 16 meses de edad, siendo uno de los casos ocurridos más tempranamente: el tamaño de este tumor fue de $20 \mathrm{cmts}$. y presentó implantaciones en el momento. Se ha relatado un caso de teratoma quístico abscedado y fistulizado a vejiga (29). La mayoría de las veces el tumor suele ser asintomático, descubriéndose casualmente al efectuar una exploración clínica, al examinar una radiografía, al ocurrir una complicación con torsión o ruptura, o con ocasión de una intervención quirúrgica motivada por causa diferente. Para una minuciosa información respecto a las características de los teratomas del ovario remitimos a la excelente revisión hecha por Woodruff, Protos y Peterson (28).
El disgerminoma es un tumor compuesto exclusivamente por células germinales indiferenciadas, que no llegan a constituir formaciones embrionarias - extraembrionarias. Aunque generalmente es unilateral, en el 5 a $10 \%$ de los casos afecta ambos ovarios. En nuestra serie de 6 tumores, dos de ellos fueron bilaterales y llegaron en estado muy avanzado, siendo logrado el diagnóstico en uno de ellos mediante biopsia de ganglio cervical metastásico. Una de las pacientes fue operada con diagnóstico de apendicitis aguda, encontrándose un tumor con torsión del pedículo. Otra, sometida a tratamiento conservador, se embarazó y tuvo un hijo normal dos años después de la extirpación del tumor. El tamaño de las neoplasias varió entre 15 y 22 cmts. Invariablemente, a excepción de una paciente de 59 años, fueron encontrados por debajo de los 20 años y no se presentaron evidencias de estados intersexuales asociados. El tratamiento preconizado es la ovariectomía unilateral para los tumores unilaterales, rodeados de una cápsula lisa y sin zonas hemorrágicas en la superficie de corte. Cuando el tumor es bilateral y hay signos de malignidad, el pronóstico es malo a pesar de una extirpación minuciosa de los órganos pélvicos; este tumor es muy sensible a la radiación pero es preferible esperar signos de recidiva para aplicarla (4).

Encontramos dos casos de estruma ovárico, el primero en una mujer de 50 años y el segundo en una de 38, asociado con elementos teratomatosos embrionarios maduros. Ambos fueron histológicamente benignos y no manifestaron signos de tireotoxicosis. Aunque el examen microscópico ha demostrado que del 5 al $20 \%$ de los quistes dermoides contienen tejido tiroideo, el término estruma sólo se aplica con propiedad a aquellos casos 
CUADRO NN 5

TUMORES DEL OVARIO

TUMORES DERIVADOS DEL ESTROMA GONADAL

CARACTERISTICAS DE LOS TUMORES DE LA TECA-GRANULOSA

\begin{tabular}{|c|c|c|c|c|c|c|c|}
\hline \multicolumn{2}{|c|}{ Paciente } & \multirow{2}{*}{$\frac{\text { Edad }}{50}$} & \multirow{2}{*}{$\begin{array}{l}\text { Localización } \\
\text { derecho }\end{array}$} & \multirow{2}{*}{$\frac{\text { Tamaño cmts. }}{11}$} & \multirow{2}{*}{$\begin{array}{c}\text { Sangrado } \\
-\end{array}$} & \multirow{2}{*}{$\begin{array}{c}\begin{array}{c}\text { Síntomas } \\
\text { Dolor }\end{array} \\
+\end{array}$} & \multirow{2}{*}{$\frac{\text { Masa }}{+}$} \\
\hline 1 & V. S. & & & & & & \\
\hline 2 & O. P. & 62 & derecho & 12 & + & - & $\div$ \\
\hline 3 & E. C. & 58 & izquierdo & 17 & + & - & + \\
\hline 4 & C. de P. & 32 & izquierdo & 6 & - & - & + \\
\hline 5 & M. G. & 56 & derecho & - & - & + & + \\
\hline 6 & O. de R. & 25 & izquierdo & 19 & - & + & + \\
\hline 7 & A. D. & 41 & izquierdo & 20 & - & - & + \\
\hline 8 & R. $M$. & 45 & derecho & 4 & - & - & + \\
\hline 9 & G. de R. & 54 & izquierdo & 7 & + & - & + \\
\hline 10 & S. P. & 54 & izquierdo & 12 & + & - & + \\
\hline 11 & T. de B. & 35 & derecho & 15 & + & - & + \\
\hline
\end{tabular}

en que dicho tejido constituye todo el teratoma o su mayor parte.

No encontramos casos de gonadoblastoma, corioepitelioma, carcinoma embrionario, teratomas embrionarios inmaduros, ni carcinoide (argentafinoma).

\section{Tumores del estroma gonadal específico}

Aunque se han descrito tumores de la granulosa puros, o formados exclusivamente por células tecales, lo común es considerarlos como formados por un componente mixto granulosotecal, aunque con predominio de un determinado tipo celular, pudiendo tener diversos grados de luteinización, por lo cual también han sido denominados tumores gránulo-tecales luteinizados.

Encontramos 11 tumores de este grupo correspondiendo al $2.9 \%$ de las neoplasias estudiadas ( 9 de la granulosa, 1 tecoma y uno mixto). La edad de las pacientes osciló entre 25 y 62 años, encontrándose 5 de ellas en postmenopausia y las restantes en plena vida reproductora. En cuanto a la paridad, varió entre grandes multíparas y alguna esterilidad primaria. Los principales síntomas se relacionaron con presencia de masa anexial y alteraciones menstruales (amenorrea, metrorragias y sangrado postmenopáusico); encontramos asociación con adenocarcinoma de endometrio en una paciente de 62 años, grávida 1 y afecta ,además de prolapso genital (7). Schroeder en 1922 fue el primero en reportar la asociación de tumores ováricos feminizantes, particularmente tecomas y tumores de las células granulosas, con adenocarcinomas de endometrio; varios autores encuentran que el riesgo aumenta después de la menopausia (11).

Se encontraron diversos tipos de hiperplasia del endometrio asociada (4 casos), y en uno, reacción decidual (Huguesdon ha señalado la posible producción de progesterona o sus derivados metabólicos en estos tumores y afirma que muchas neoplasias de células lipoideas no son sino tecomas luteinizados). A la paciente número 6 , de 25 años de edad, se le descubrió un tecoma intervenido a los 17 días 
del puerperio de su quinto parto, y se acompañaba de ascitis.

El tamaño de los tumores varió entre 4 y 20 centímetros y la gran mayoría de ellos presentaban zonas quísticas entremezcladas con elementos sólidos; no tuvimos casos bilaterales.

Respecto a la conducta se considera que estas neoplasias deben ser tratadas con salpingo-ovariectomía unilateral en mujeres jóvenes con tumores encapsulados, sin ascitis ni adherencias, reservando el tratamiento radical para las postmenopáusicas, 0 en las que no se desee preservar la función reproductora (1).

No encontramos ningún caso de arrenoblastoma, tumor raro, generalmente unilateral, que alcanza un tamaño de 10 a 12 centímetros, usualmente productor de andrógenos con sus consecuencias (desfeminización y virilización), y de dudosa malignidad.

\section{Tumores de restos embrionarios}

Los tumores de Brenner, de los cuales hallamos tres casos, a menudo se confunden con fibromas en el examen macroscópico, aunque por lo general son de menor tamaño (10 a 15 centímetros), menos nodulares y de consistencia más gomosa. El tumor es casi siempre unilateral y por lo general benigno. Farrar y Green (10) describen dos casos de bilateralidad, al igual de Kretchmar y colaboradores (14). El mayor número se encuentra en pacientes por encima de los 50 años. En los hallados en esta revisión, con tamaños de 5 a $20 \mathrm{cms}$. las edades respectivas fueron 58,22 y 34 años. El último de ellos considerado histológicamente maligno y a pesar de un tratamiento radical (anexohisterectomía y cobaltoterapia postoperatoria en el Instituto Nacional de Cancerología), su comportamiento fue tan agresivo que determinó la muerte de la paciente en el término de tres meses. En la última década, en una $r \in v i s i o ́ n$ reciente de Idelson se han señalado 26 casos de tumores malignos de Brenner (19). Aunque antes se creía que la mayor parte de estos tumores eran inertes, cada vez parece más claro que algunos son activos en el aspecto hormonal.

En cuanto a otros tumores raros (Mesonefroma, mesometanefroma, mesotelioma (20), luteoma, restos adrenales, células hiliares, etc.), su histogénesis es dudosa y a causa de su rareza son menos interesantes para el clínico. No encontramos ninguno de ellos.

\section{Tumores del estroma inespecífico}

El fibroma del ovario puede alcanzar gran tamaño porque no interfiere con las estructuras vecinas y por consiguiente es asintomático. En nuestra serie aparecieron 8 tumores, invariablemente unilaterales y benignos, $\mathrm{cu}$ yos tamaños extremos alcanzaron $6 y$ 17 centímetros. Las principales manifestaciones fueron tumor pélvico, dolor moderado, y en tres de ellos ascitis, pero sin configurarse en ninguno la tríada característica del síndrome de Meigs. Las edades extremas fueron de 36 y 52 años, con mayor incidencia en la cuarta década de la vida. Histológicamente son similares al fibroadenoma común de la glándula mamaria y en ocasiones sufren transformación quística, alcanzando tamaños grandes, y llamándoseles citadenofibromas. El fibroma puede extirparse de un solo lado, pero como se presenta en mujeres de edad avanzada el tratamiento más racional es la salpingo-ovariectomía bilateral e histerectomía.

Woodruff, Castillo y Novak (27) recopilaron 35 casos de linfoma del ovario, pero sólo en 9 de ellos la evi- 
dencia fue suficiente para catalogarlos como tumores primarios.

\section{Tumores metastásicos}

Aunque seguramente su incidencia sea mayor cuando existen neoplasias avanzadas de otros órganos, la ocurrencia fue exigua y se limitó a un tumor de Krukenberg, reportado anteriormente por Duarte Contreras y Luzardo (6), en una mujer, de 40 años, grávida 2, para 2, quien dos años después de una gastrectomía por adenocarcinoma gástrico, presentó tumoración pélvica bilateral de los ovarios tratada mediante histerectomía y salpingo ovariectomía bilateral; la paciente falleció un año después con metástasis pulmonares. La revisión de la literatura revela 38 casos reportados como tumor de Krukenberg primario, pero de éstos sólo se aceptan 18 casos, uno de ellos informado en Colombia por Castillo y cols. (2), porque cumplen a satisfacción los postulados de Novak y Woodruff.

\section{Patología asociada}

\section{CUADRO Nㄴ 6}

TUMORES DEL OVARIO PATOLOGIA ASOCIADA

\begin{tabular}{lc}
\hline Entidad & No Casos \\
\hline Fibroma uterino & 29 \\
Salpingitis crónica & 28 \\
Prolapso genital & 13 \\
Quistes foliculares & 10 \\
Polimicroquistosis ovárica & 6 \\
Embarazo tubárico & 5 \\
Colelitiasis & 5 \\
Hidrosálpinx & 5 \\
Pólipo endometrial & 4 \\
Hiperplasia endometrial & 4 \\
Piosalpinx & 4 \\
Adenocarcinoma de endometrio & 2 \\
\hline
\end{tabular}

Es interesante consignar que muchos tumores del ovario fueron hallazgo ocasional en pacientes que se operaron por otra entidad dependiente del aparato genital, y también en laparotomías por enfermedades quirúrgicas del tracto gastrointestinal y vías biliares, por lo cual es recomendable una cuidadosa revisión de los órganos pélvicos en estas intervenciones.

\section{Conclusiones}

1. Debe practicarse incisión exploratoria de la pelvis en toda tumoración pelviana que aparezca después de la menopausia; en una mujer de cualquier edad con agrandamiento anexial que mantenido bajo observación haya crecido más de $20 \mathrm{cmts.;}$ en toda tumoración anexial de $10 \mathrm{cmts}$. o más de diámetro, o en la incapacidad de determinar si se trata de un fibromioma o un quiste del ovario.

2. La alta mortalidad y la dificultad en el diagnóstico temprano del carcinoma ovárico, nos muestran como único enfoque alternativo las medidas profilácticas; nos asociamos en apoyar el examen bimanual periódico de toda mujer por encima de los 30 años, con el propósito de lograr detección temprana y desde luego, mejor pronóstico $(11,29)$.

3. Los ovarios deben explorarse, y si es necesario, someterse a biopsia, al efectuar cualquier laparotomía en la mujer, y deben extirparse profilácticamente en los casos de cáncer de estómago, intestino, y en el carcinoma avanzado de la glándula mamaria.

4. Aunque muchos autores (clínicos y patólogos) propugnan la conservación de los ovarios en casos de histerectomía por otras condiciones benignas, es necesario tener en cuenta los siguientes hechos: la bilateralidad de los tumores ováricos que en algunos casos llegan hasta el 37\%, posible presencia de tumores ocultos, algunos de carácter letal que podrían haberse prevenido mediante la extirpación previa de los ovarios, y posibilidad 
de aparición de patología benigna 0 maligna en los ovarios residuales. $\mathrm{Na}$ turalmente en la mujer menopáusica - postmenopáusica es indudable la conveniencia de extirpar los ovarios al practicar histerectomía.

5. El mejor tratamiento para las neoplasias del ovario es la salpingo ovariectomía bilateral con histerectomía total por vía abdominal, especialmente en la postmenopausia y en mujeres que ya han completado su familia. En las madres en potencia en quienes debe preservarse la función ovárica y en aquellas que constituyen malos riesgos quirúrgicos, I a cirugía conservadora resulta adecuada en los siguientes casos: quistes dermoides, cistadenoma pseudomucinoso unilateral, cistedenoma seroso unilateral no papilar, fibroma, tecoma, tumor de Brenner y tumores carnosos unilaterales con cápsula intacta y ausencia de zonas hemorrágicas (tumores de células granulosas y disgerminoma ).

\section{Resumen}

Se hace un estudio de revisión de las neoplasias ováricas ocurridas en un lapso de 13 años en el Hospital San Juan de Dios, de Cúcuta. Se recopilaron 367 casos de los cuales 317 $(86.9 \%)$ fueron benignos y 50 ( $13.1 \%)$ malignos. Las edades extremas oscilaron entre 3 y 90 años. Siguiendo la clasificación histogenética de Hertig y Gore, el tipo más frecuente de tumor fue el cistadenoma seroso, seguido de los quistes dermoides, cistadenocarcinoma y diversas clases de tumores. Se relievan la baja incidencia de tumores pseudomucinosos, la coexistencia de un cistadenocarcinoma papilar con un tumor de células granulosas, la asociación de un cistedon carcinoma seroso con embarazo y el hallazgo de un tumor de Brenner maligno. Se hace énfasis sobre el aumento de la incidencia del carcinoma ovárico, la baja tasa de supervivencia a los 5 años y la necesidad de medidas profilácticas para su detección temprana.

\section{BIBLIOGRAFIA}

1 BELTRAN S. R. y cols. Tumores feminizantes del ovario. Ginec. Obstet. Méx. 32: 43, 1972.

2 CASTILLO, J. y cols. Tumor de Krukenberg primario del ovario. Rev. Col. Obst. Ginec. 24: $218,1973$.

3 CERON, E. A. y cols. Arrenoblastoma. Ginec. Obstet. Méx. 33: 313, 1973.

4 CORSCADEN, GUSBERG, S. B. Y FRICK, H. C. Cáncer Ginecológico. Ed. Médica Panamericana. Buenos Aires, 4ad. Ed. 1971.

5 DARNALT, R. E. y cols. Tumor ovárico gigante. Rev. Col. Obst. Ginec. 23: 133, 1972.

6 DUARTE CONTRERAS. A. y LUZARDO L. F. Tumor de Krukenberg del ovario. Rev. Col. Obst. Ginec. 25: 13, 1974.

7 DUARTE CONTRERAS, A., ZUÑIGA G. R: Y URIBE B. G. Tumor de la granulosa asociado a adenocarcinoma de endometrio. Rev. Col. Obst. Ginec. 19: 111, 1968.

8 ERMINY A. y cols. Tumores malignos primitivos del ovario. Rev. Obst. Ginec. Venez. 30: $319,1970$.

9 FARRAR, H. K., BYRAN, R. Equilateral distribution of ovarian tumors. Am. J. Obst. Ginec. 80: 1085. 1960.

10 FARRAR, H. K., GREENE, R. R. Bilateral Brenner tumors of the ovary. Am. J. Obst. Gynec. 80: 1089, 1960.

11 GRABER, E. A. Diagnóstico temprano del carcinoma ovárico. Clínicas Obstét. Ginecol. Norte América. Ed. Interamericana S. A. México, 958, 1969.

12 JANOVSKI, N., PARAMANANDHAN, T. L. Ovarian tumors. W. B. Saunders Company. Philadelphia. 1973.

13 KOTTMEIER, L. y cols. Carcinoma de ovario y su tratamiento. Progresos de la Ginecología. Meigs y Sturgis. Ed. Científico Médica. Barcelona. 4: 397, 1972. 
14 KRETCHMAR, L. y cols. Bilateral Brenner tumors. Am. J. Obst. Gynec. 82: 389, 1961.

15 LEE, A. B., BOWERS, P. Bilateral primary ovarian carcinomas. Am. J. Obst. Gynec. $80: 1083,1960$.

16 LONG, M. E. y SOMMERS, S. C. Clasificación por períodos y grados, e histoquímica, de tumores epiteliales ováricos. Clín. Obstét. Ginecol. Norte Amér. 937, 1969.

17 MORAN, J. P. and MILLERICK, J. D. Ten years experience with ovarian malignancy at a United States naval hospital. Am. J. Obst. Gynec. 82: 375, 1961.

18 NOGUCHI, T. and LONSER, E. Unusual teratoma of the ovary with implantation in the abdominal cavity. Am. J. Obst. Gynec. 82: $381,1961$.

19 NOVAK, E. R., SEEGAR, J. G. y JONES, H Ginecología. Nueva Editorial Interamericana. México. 8ad. Ed. 1971.

20 PARMLEY, T. H. and WOODRUFF, J. D. The ovarian mesothelioma. Am. J. Obst. Gynec. 120: 234, 1974.

21 PARSONS, L. and SOMMERS, S. C. Gynecology. W. B. Saunders Co. Philadelphia. 1961.
22 RODRIGUEZ, A. J. y RICO N. P. Influencia de los tumores ováricos sobre el endometrio. Ginec. Obstet. Méx. 31: 583, 1972.

23 SANCHEZ, T. F. Historia de la Obstetricia y la Ginecología en Latinoamérica. FLASOG. Imprenta Distrital de Bogotá, Pág. 139, 1970.

24 SCULLY, R. E. Tumores ováricos originados a partir de las células germinales. Progresos de la Gniecología. Meigs y Sturgis. Edit. Científico Médica. Barcelona. 4: 407, 1971.

25 VALENZUELA, S. y cols. Revisión de 492 casos de neoplasias ováricas estudiadas en un período de 11 años. Ginec. Obstet. Méx. 33 : $125,1973$.

26 WADE, M. E., JANOVSKI, N. A. and BYSSHE, S. M. Pseudomucinous cistadenocarcinoma associated with pregnancy. Am. J. Obst. Gynec. 85 : 919, 1963.

27 WOODRUFF, J. D., NOLI, C. R. D. and NOVAK, E. R. Limphoma of the ovary. Am. J. Obst. Gynec. 85: 912, 1963.

28 WOODRUFF, J. L., PROTOS, P. and PETERSON, W. F. Ovarian Teratomas. Am. J. Obst. Gynec. 102: 702, 1968.

29 ZARIN, G. F. Teratoma quístico del ovario con absceso y fístula a vejiga. Ginec. Obstet. Méx. 36: 49, 1974. 